\title{
M/L of X-ray luminous clusters from weak lensing
}

\author{
H. Dahle \\ Institute of Theoretical Astrophysics, University of Oslo, \\ P.O. Box 1029, Blindern, N-0315 Oslo, Norway email: hdahle@astro.uio.no
}

\begin{abstract}
We present results from a weak lensing survey of a sample of 53 clusters of galaxies at $0.15<z<0.4$, selected based on their high X-ray luminosities. For each cluster, we measure the mass-to-light ratio out to a radius $R \sim(0.5-1.5) h^{-1} \mathrm{Mpc}$. By measuring the lensing masses of a large sample of clusters in a consistent way, we quantify the intrinsic scatter in $M / L$ and determine its average value for massive clusters. In the near future, an ongoing spectrophotometric and weak lensing survey of a supercluster region in Aquarius will extend $M / L$ measurements to much larger radii and explore its dependence on scale and environment.
\end{abstract}

\section{Introduction}

Gravitational lensing measurements based on deep optical or near-IR imaging provides the most direct way of determining the mass-to-light ratios of galaxy clusters. Previous results based on weak lensing studies of clusters (see e.g. the compilation by Mellier 2002) show a wide scatter in $M / L$ values, but it is uncertain whether this reflects a true scatter in $M / L$ or whether it is mostly caused by differences in reduction and analysis techniques, assumptions about source galaxy distances, different passbands being used etc. On the other hand, several weak lensing studies have also revealed a few "dark cluster" candidates that have been detected based on their gravitational lensing effect and which appear to have extreme $M / L$ values compared to normal clusters. At least one reported "dark cluster" candidate has a measured bolometric mass-to-light ratio $M / L_{\text {bol }} \geqslant 1000 h$ in solar units (Erben et al. 2000; Gray et al. 2001).

By analyzing weak lensing data of a large sample of clusters in a consistent way, it should be possible to accurately determine $M / L$ at the high-mass end of the cluster mass function and also quantify the intrinsic scatter in $M / L$.

\section{X-ray luminous cluster sample}

Weak gravitational lensing measurements of $38 \mathrm{X}$-ray luminous galaxy clusters were presented by Dahle et al. (2002), and subsequent data have been obtained for 15 additional clusters with similar selection criteria. Clusters in the redshift range $0.1<z<0.4$ were selected from different ROSAT-based cluster surveys (Briel \& Henry 1993; Ebeling et al. 1996, 1998, 2000; Böhringer et al. 2000), and almost all targets have X-ray luminosity $L_{\mathrm{X}, 0.1-2.4 \mathrm{keV}} \geqslant 10^{45} \mathrm{erg} \mathrm{s}^{-1}$. A subsample of 12 clusters were observed using the UH8K camera at the University of Hawaii $2.2 \mathrm{~m}$ telescope (covering a field $\sim 3 h^{-1} \mathrm{Mpc}$ across at the distance of the clusters), while the rest were observed with $2 \mathrm{k}$ CCD cameras at the UH $2.2 \mathrm{~m}$ telescope and at the Nordic Optical Telescope (covering smaller fields of $\sim 1 h^{-1} \mathrm{Mpc}$ ). Details on the reduction and weak lensing analysis of this data set are provided by Dahle et al. (2002). 


\section{Mass-to-light ratio vs. X-ray luminosity}

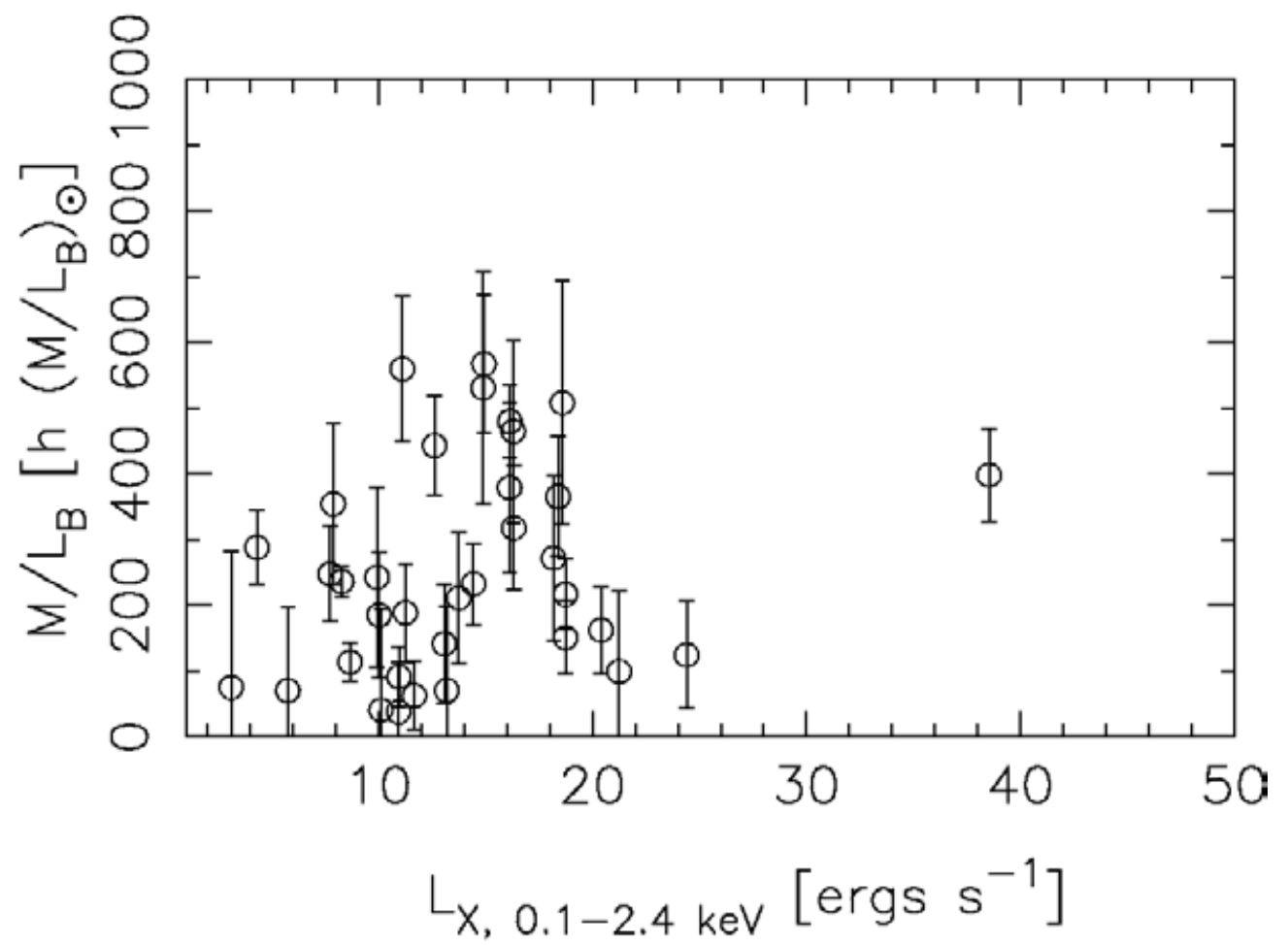

Figure 1. B-band mass-to-light ratio for 35 clusters in our X-ray luminous sample as a function of X-ray luminosity.

\subsection{Measured and predicted surface mass density}

The shear estimator introduced by Kaiser (2000) was used to measure the weak gravitational shear. The shear inversion techniques of Kaiser \& Squires (1993) and Squires \& Kaiser (1996) were used to reconstruct the projected dimensionless mass surface density $\kappa_{M}$ from UH8K and $2 \mathrm{k}$ CCD data, respectively. We generated sets of source galaxy catalogues with randomized shear, keeping the galaxy positions as in the real catalogues, while randomly shuffling the measured galaxy shapes, such that the spread of values in the ensemble of resulting mass maps could be used to evaluate the uncertainties in the mass maps.

Assuming a mass-traces-light model for the clusters, a predicted $\kappa_{L}$ for the projected mass density was generated based on $I$-band photometry. In an image of $\kappa_{L}$ with a pixel size corresponding to a solid angle $d \Omega$, the contribution of a galaxy of apparent magnitude $m_{I}$ and redshift $z$ to the pixel on which the galaxy is located, is given by:

$$
\kappa_{L} d \Omega=\frac{M}{L_{B}} \frac{M_{\odot}}{(10 \mathrm{pc})^{2}} \frac{(1+z)^{4}}{\Sigma_{\text {crit }}(z)} 10^{0.4\left[M_{B} \odot-m_{I}-K_{B I}(z)\right]},
$$

where $M / L_{B}$ is the rest-frame $B$-band mass-to-light ratio (in solar units) used for the prediction, and $\Sigma_{\text {crit }}(z)$ is the effective critical density (where the distribution of 
background galaxy redshifts is estimated from HDF-based photometric redshifts). The quantity $K_{B I}(z)$ combines the conventional $K$-correction and the rest-frame $B-I$ color. It is possible to reliably select early-type galaxies and estimate their redshifts based on their $V-I$ colors (Wilson et al. 2001), but we initially adopted a different approach, assuming that the observed galaxy flux in the fields was contributed by galaxies at the cluster redshift plus a background with constant surface flux density. A few bright galaxies that were obvious foreground objects were removed by hand.

A radially dependent correction for the contamination by cluster galaxies in our source galaxy catalogue was calculated, and the $\kappa_{L}$ maps were corrected accordingly, to mimic the corresponding effect on the $\kappa_{M}$ maps.

\subsection{Results for $M / L$}

The rest-frame B-band mass-to-light ratios in the cluster fields were calculated by taking the discrete Fourier transform of the predicted and measured surface density images and comparing the amplitudes of the Fourier modes. In Figure 1, the resulting values for $M / L_{\mathrm{B}}$ are plotted against X-ray luminosity. The $M / L$ values were calculated for a concordance model universe with $\left(\Omega_{\Lambda}, \Omega_{m}\right)=(0.7,0.3)$.

From a subset of 35 clusters, we find $\left\langle M / L_{B}\right\rangle=(219 \pm 11) h\left(M / L_{B}\right)_{\odot}$, and a median value of $M / L_{B}=236 h\left(M / L_{B}\right)_{\odot}$. Taken at face value, the spread of observed $M / L$ values indicate an intrinsic scatter in $M / L_{B}$ of about $\sigma\left(M / L_{B}\right)=118 \pm 19$ about the mean value. However, part of this scatter could be due to incomplete removal of foreground/background contamination in the galaxy catalogues, and our underlying assumption that mass traces light may not be valid for all systems. Since our data indicate a significant intrinsic cluster-to-cluster variation in $M / L$, this may at least partly explain the wide range of cluster $M / L$ values reported in the literature.

The halo-mass dependence of $M / L$ is still not a fully resolved issue: While some dynamical studies indicate that mass increases faster than luminosity (Girardi et al. 2002), such a trend is not seen from a recent X-ray based study (Sanderson \& Ponman 2003). We note that since our clusters only cover a modest range in mass, our results must be combined with other data sets probing less massive systems to be able to address this issue.

\section{Survey of the Aquarius supercluster region}

The highest projected density of $R \geqslant 1 \mathrm{Abell} / \mathrm{ACO}$ clusters in the sky is located around $\alpha=23.2^{h}, \delta=-22 \mathrm{deg}$ (Abell 1961). This high density is caused by two superclusters (at $z=0.08$ and $z=0.11$ ) seen in projection, along with a filament extending outwards along the line of sight from the far end of the $z=0.11$ supercluster.

A combination of weak lensing data with galaxy spectroscopy and photometry allows to study the relationship between mass and light in this region, in particular the scaleand type-dependence of galaxy bias. An ongoing survey combines galaxy spectroscopy with the $2 \mathrm{dF}$ instrument at the $3.9 \mathrm{~m}$ AAT with a weak-lensing survey based on wide-field imaging from the WFI at the ESO $2.2 \mathrm{~m}$ telescope. In a total of 20 WFI pointings we cover an area of 6.2 square degrees (corresponding to a transverse scale $\sim 20 h^{-1} \mathrm{Mpc}$ at the supercluster redshift), containing 25 previously known galaxy clusters. We have obtained spectra of $\sim 3000$ galaxies down to $R=19.5$ in this region and use this data to identify 26 previously unknown clusters and groups and estimate their redshifts and velocity dispersions along with the known clusters (Smith et al. 2004). Our results indicate that the $z=0.08$ and $z=0.11$ superclusters in Aquarius are both very massive systems, comparable to the Shapley supercluster or the Corona Borealis supercluster. The imaging 
KS93 $\kappa(r)$ RECONSTRUCTION OF AQR_SC_1

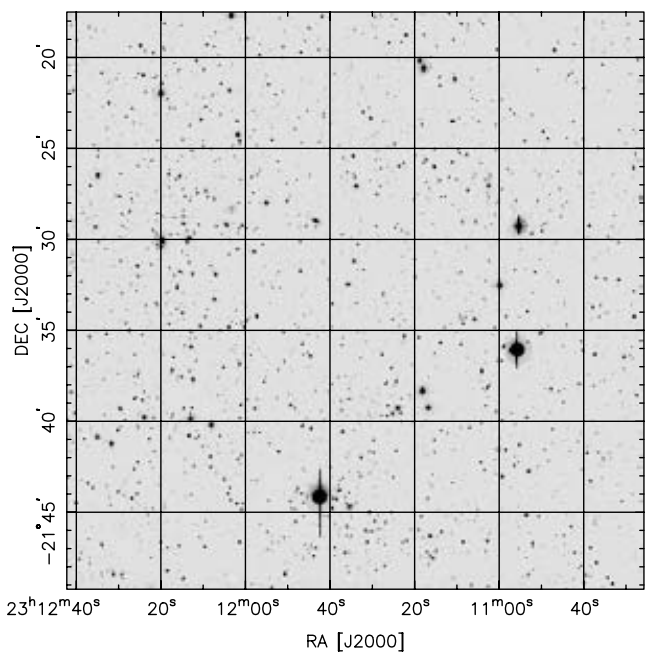

PREDICTED $\kappa(r)$ FROM ALL GALAXIES

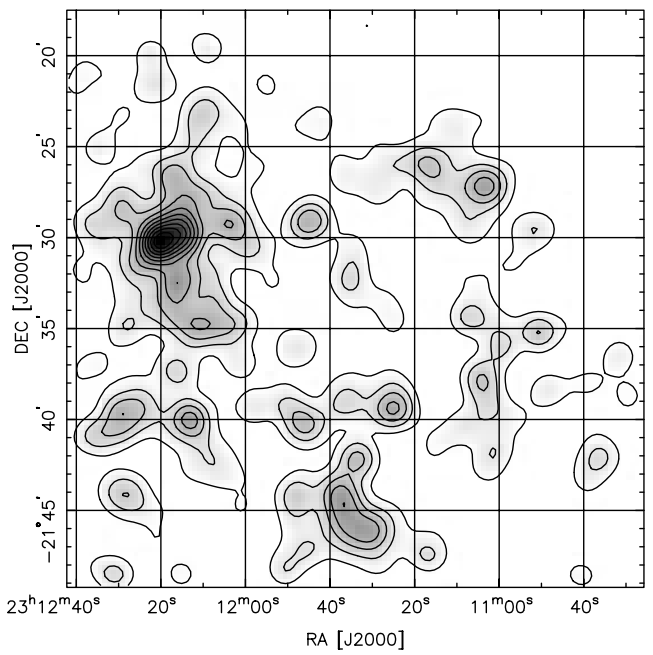

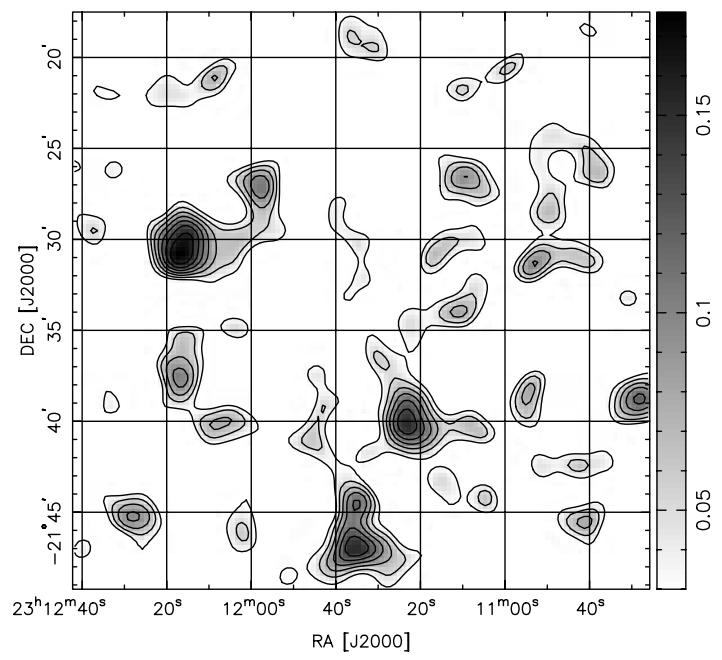

GALAXY NUMBER DENSITY

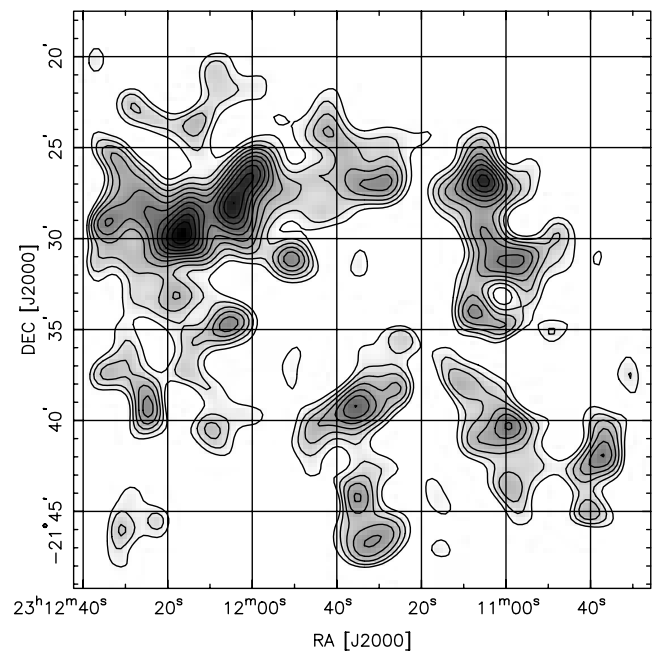

Figure 2. Preliminary results for 1 of the 20 pointings within the Aquarius supercluster region. Top left: $R$-band image based on ESO WFI imaging. Top right: The surface mass density $\kappa_{M}$, determined from weak shear measurements of background galaxies. Bottom left: Predicted surface mass density associated with the light from galaxies in the field, $\kappa_{L}$. To generate this plot, the galaxies were all assumed to lie at a single redshift, but in our ongoing analysis we use $B-R$ color information combined with our $2 \mathrm{dF}$ spectroscopic redshifts to generate a more accurate measurement of $\kappa_{L}$. Bottom right: The number density of all galaxies in the field. Two Abell clusters are located within this field, A2554 ( $z=0.111$; upper left) and A2550 ( $z=0.122$; bottom center), in addition to several lesser clusters and galaxy groups.

part of this survey is still ongoing, and preliminary results for one of the 20 WFI pointings is shown in Figure 2. Several peaks that correspond to previously known clusters are visible in the maps, along with some new systems.

\section{Acknowledgements}

I thank my collaborators R. E. Smith, P. B. Lilje and S. J. Maddox. 


\section{References}

Abell, G. O., 1961, AJ, 66, 607

Böhringer, H. et al. 2000, ApJS, 129, 435

Briel, U., \& Henry, J. P. 1993, A\& A, 278, 379

Dahle, H., Kaiser, N., Irgens, R. J., Lilje, P. B., \& Maddox, S. J. 2002, ApJS, 139, 313

Ebeling, H., Voges, W., Böhringer, H., Edge, A.C., Huchra, J. P. \& Briel, U.G. 1996, MNRAS, 281,799

Ebeling, H., Edge, A. C., Böhringer, H., Allen, S., Crawford, C. S., Fabian, A.C., Voges, W., \& Huchra, J. P. 1998, MNRAS, 301, 881

Ebeling, H., Edge, A. C., Allen, S. W., Crawford, C. S., Fabian, A. C., \& Huchra, J. P. 2000, MNRAS, 318, 333

Erben, T., van Waerbeke, L., Mellier, Y., Schneider, P., Cuillandre, J.-C., Castander, F. J. , \& Dantel-Fort, M. 2000, A\& A, 355, 23

Girardi, M., Manzato, P., Mezzetti, M., Giuricin, G., \& Limboz, F. 2002, ApJ, 569, 720

Gray, M. E., Ellis, R. S., Lewis, J. R., McMahon, R. G., \& Firth, A. E. 2001, MNRAS, 325, 111

Kaiser, N. 2000, ApJ , 537, 555

Kaiser, N. \& Squires,G. 1993, ApJ, 404, 441, KS93

Mellier, Y. 2002, Space Science Reviews, 100, 73

Sanderson, A. J. R. \& Ponman, T. J. 2003, MNRAS, 345, 1241

Smith, R. E., Dahle, H., Maddox, S. J., \& Lilje, P. B. 2004, astro-ph/0401641

Squires, G., \& Kaiser, N., 1996, ApJ, 473, 65

Wilson, G., Kaiser, N., Luppino, G. A., \& Cowie, L. L. 2001, ApJ, 555, 572 